\section{Inoperable Lebermetastasen: Ablation plus Chemotherapie}

\begin{abstract}
Patienten mit einem in die Leber metastasierten kolorektalen Karzinom (CRC) erhalten standardmäßig eine systemische Therapie. Profitieren die Patienten möglicherweise von einer zusätzlichen Radiofrequenzablation der Lebermetastasen.
\end{abstract}

Etor wa $50 \%$ aller Patienten mit einem kolorektalen Karzinom (CRC) entwickeln früher oder später Metastasen in der Leber. Ist eine Resektion möglich, beträgt die 5-Jahres-Gesamtüberlebensrate rund $50 \%$. Chemotherapie allein als führt zu einem medianen Überleben von etwa zwei Jahren. Um dies zu verbessern, wurden in der letzten Dekade verschiedene Techniken zur lokalen Tumordestruktion entwickelt, darunter die Radiofrequenzablation. Bisher lagen keine validen Daten zur Effektivität dieser Therapieoption beim nicht resektablen ausschließlich in die Leber metastasierten CRC vor.

In der randomisierten EORTC-Intergroup-Studie 40004 wurde der mögli- chen Nutzens einer Radiofrequenzablation (RFA) für diese Patienten untersucht. 119 Patienten wurden in zwei Therapiegruppen randomisiert: Alle Patienten erhielten als systemische Therapie ein FOLFOX-Regime (5-Fluorouracil/ Folinsäure/Oxaliplatin) ggf. in Kombination mit Bevacizumab; Guppe 1 ausschließlich, in Gruppe 2 wurde zusätzlich eine RFA der Lebermetastasen durchgeführt. Primäres Studienziel war das Gesamtüberleben der Patienten im Kombinationsarm nach 30 Monaten von über $38 \%$.

Der definierte primäre Endpunkt wurde erreicht: nach 30 Monaten lebten im Kombinationsarm noch $61,7 \%$ der Patienten. Für die Forscher überra- schend war die Gesamtüberlebensrate war mit $57,6 \%$ in Gruppe 1 jedoch nur unwesentlich niedriger. Das mediane 3-Jahres-Gesamtüberleben betrug für Gruppe $140,5 \%$ und für Gruppe 2 $45,3 \%$. Allerdings lebten in Gruppe 2 nach drei Jahren noch 27,6\% der Patienten ohne Progress, in Gruppe 1 nur $10,6 \%$.

Fazit: Patienten mit einem inoperablen hepatisch metastasierten kolorektalen Karzinom, deren Tumorlast vor der systemischen Therapie per Radiofrequenzablation verringert wurde, profitieren von einem deutlich verlängerten progressionsfreien Überleben nach drei Jahren. Unklar bleibt bislag der mögliche Benefit des kombinierten Vorgehens für das Gesamtüberleben.

Wolfgang Zimmermann

Ruers T et al. Radiofrequency ablation combined with systemic treatment versus systemic treatment alone in patients with non-resectable colorectal liver metastases: a randomized EORTC Intergroup phase II study (EORTC 40004). Ann Oncol. 2012;23(10):2619-26.

\section{Nodal-positives Analkarzinom: Vorteil durch Brachytherapie-Boosterung}

Die strahlentherapeutische Behandlung des lokal fortgeschrittenen Analkarzinoms verläuft in Europa meist zweistufig: Der initialen Radiotherapie folgt zur Dosisaufsättigung im Tumor eine weitere Strahlenbehandlung (Boosterung). Ob diese bei Lymphknotenbefall besser als externe Bestrahlung oder als Brachytherapie erfolgt, wurde retrospektiv geprüft.

A n der Studie wurden 99 Patienten mit nodal-positivem Analkarzinom ohne Fernmetastasen behandelt. 49 Studienteilnehmer hatten einen Tumor im Stadium III. Bei 67 Studienteilnehmern lag ein N1-Karzinom vor, die übrigen Patienten zeigten ein Nodalstadium N2 oder 3.

Alle Patienten hatten sich zunächst der Radio- oder Radiochemotherapie mit einer externen Bestrahlung von 45,1 Gy als medianer Dosis unterzogen. Dabei wurden 1,8 bis 2,0 Gy pro Fraktion appliziert. Zur Boosterung erfolgte in der ersten Gruppe $(n=49)$ eine externe Bestrahlung des ursprünglichen Tumor- bereichs mit Photonen oder Elektronen. Bei den Patienten der zweiten Gruppe ( $\mathrm{n}$ $=50)$ setzten die Ärzte Iridium192-Drähte mit einer Länge von 5-8 cm ein. In beiden Studienarmen lag die Dosis bei $18 \mathrm{~Gy}$.

Nach einer Beobachtungszeit von 71,5 Monaten lagen die 5-Jahres-Raten für das Auftreten von Lokalrezidiven nach der externen Boosterung bei $32 \%$, in der Gruppe mit BrachytherapieBoost jedoch nur bei $11 \%(\mathrm{p}=0,03)$. Dieser Unterschied war für Patienten im N1-Stadium mit 31 vs. $4 \%$ noch deutlicher, in der Gruppe mit Stadium N2/3 ergab sich keine signifikante Dif- ferenz. Die multivariate Analyse für die N1-Population zeigte die Brachytherapie als einzigen prognostischen Faktor für das Lokalrezidiv-Risiko.

Die 5-Jahres-Raten für das Gesamtüberleben boten mit 73,3\% in Gruppe 1 und $77,5 \%$ in Gruppe 2 keine signifikanten Unterschiede. Gleiches galt für das Kolostoma-freie-Überleben (69 vs. $74 \%)$ oder das Auftreten von Fernmetastasen (7 vs. 11 Patienten).

Fazit: Die vorliegende retrospektive Studie zeigte, dass Patienten mit lokal fortgeschritttenem Analkarzinom von einer Brachytherapie als Boosterung im Hinblick auf das Lokalrezidiv-freie Überleben profitieren. Dies gilt auch für Patienten im Stadium N1. Bei den weiteren klinischen Endpunkten ergaben sich keine Nachteile für die Brachytherapie.

Andreas Fischer

Moureau-Zabotto L et al. Role of brachytherapy in the boost management of anal carcinoma with node involvement (CORS-03 Study). Int J Radiat Oncol Biol Phys. 2013; 85(3): e135-42. 